\title{
Rursus
}

Russus

Poiétique, réception et réécriture des textes antiques

9 | 2016

Commentaires anciens (pragmatique \& rhétorique)

\section{Exercices scolaires: un moyen de réfléchir sans émotion et sans danger}

School exercises: a way of thinking without emotion and without danger

\section{Francesca Mestre}

\section{(2) OpenEdition}

Journals

Édition électronique

URL : http://journals.openedition.org/rursus/1200

DOI : $10.4000 /$ rursus. 1200

ISSN : 1951-669X

Éditeur

Université Nice-Sophia Antipolis

Référence électronique

Francesca Mestre, "Exercices scolaires: un moyen de réfléchir sans émotion et sans danger », Rursus [En ligne], 9 | 2016, mis en ligne le 29 juillet 2016, consulté le 19 avril 2019. URL : http:// journals.openedition.org/rursus/1200; DOI : 10.4000/rursus. 1200

Ce document a été généré automatiquement le 19 avril 2019

Rursus 


\section{Exercices scolaires: un moyen de réfléchir sans émotion et sans danger}

School exercises: a way of thinking without emotion and without danger

Francesca Mestre

1 Le système scolaire grec, sur lequel grosso modo, s'est aussi formée l'école romaine, est assez connu: maintes allusions aux maîtres et à l'apprentissage sont répérables dans les textes littéraires qui nous sont parvenus, en grec et en latin, ce qui fait que de très importantes études sur l'éducation dans l'antiquité ont été réalisées par des spécialistes ; dans ce domaine, le livre déjà classique de Marrou a marqué un tournant vers le milieu du XXe siècle. Ensuite, la découverte, la lecture et l'analyse d'un bon nombre de papyrus a permis, sans doute, de compter avec des témoignages directs de la structure de l'éducation scolaire chez les anciens et son application concrète -les études concernant les exemplaires d'exercices scolaires qui ont été menées par R. Cribiore ou T. Morgan ${ }^{1}$, entre autres, en sont un bon exemple. En outre, du point de vue de la pragmatique que l'école des anciens envisageait au niveau secondaire et supérieur, nous possédons les traités de progymnasmata qui nous sont précieux pour comprendre comment l'école bâtissait ses instruments et ses outils, d'une part, et, de l'autre, pour essayer d'établir jusqu'à quel point, et par quels moyens, elle reste présente au sein d'un bon nombre de productions textuelles, non-scolaires, de l'antiquité, surtout de l'antiquité post-classique, et notamment de la période impériale et jusqu'à la fin de l'antiquité -et même au delà-, puisque c'est à cette époque-là que le système scolaire est déjà complètement fixé2

Je me propose ici de montrer que les pratiques lettrées du monde gréco-romain de l'Empire -et même avant-reposent, au delà des murs de l'école, sur les exercices scolaires de la paideia rhétorique. Et pour ce faire, il faudra tenir compte des traités de progymnasmata et des données théoriques esquissées par leurs auteurs en vue d'appuyer l'élaboration d'un discours quelconque, au moyen de la mise en oeuvre de quelques-uns de ces progymnasmata ainsi que des exercices d'accompagnement, et des possibilités que leur pratique conjointe offre à la composition. En outre, certains textes en latin, quelque 
peu différents des textes grecs, seront indispensables pour envisager de quelle façon la tradition grecque a été conservée et adaptée aux besoins et aux goûts plus particulièrement romains ${ }^{3}$.

Mais pourquoi aborder le sujet des progymnasmata dans le contexte d'une enquête sur le commentaire et sa pragmatique? Je ne vais pas, évidemment, développer en détail les différents aspects de chaque progymnasma, de leur pragmatique à eux -il me faut, sur ce point, renvoyer à quelques études ${ }^{4}$ et, évidemment, aux Traités qui nous sont parvenus, dont je cite en bibliographie éditions et traductions-; j'essaierai pourtant de montrer que la démarche formelle de ces exercices scolaires, dans leur conception et leur mise en place, est voisine de la pragmatique du commentaire; plus encore : dans la plupart des cas, les exemples de chacun des progymnasmata que nous proposent Théon ou, surtout, Aphthonios sont enracinés dans l'idée du commentaire, bien plus que sur la répétition stricte des sources anciennes, autrement dit, le commentaire comme forme, qui vise à évoquer, rapprocher et remanier les anciens textes, est toujours à l'arrière-plan dans les progymnasmata. Donc, si la pragmatique des progymnasmata est voisine de celle du commentaire et permet la comparaison avec celle-ci, il est vrai aussi que les progymnasmata participent d'une certaine façon des commentaires sur la tradition qui sont à la portée des érudits et des écoles depuis l'époque hellénistique, et que leur influence sur les formes discursives de l'élite ainsi que leur formalisation en traités montre une possible interdépendance implicite entre commentaire et progymnasmata.

\section{Les progymnasmata d'après les traités grecs}

4 En effet, nous conservons cinq Traités de progymnasmata, que nous pouvons dater entre le Ier et IVe siècles. Leur lecture est loin d'être un plaisir -il y en a même, d'après Kennedy, qui sont mal écrits ${ }^{5}$ - mais il faut avouer qu'ils sont une source majeure pour comprendre l'éducation: les outils qu'elle utilise, les buts qu'elle se propose, les valeurs qu'elle souligne. Les Traités font une description de la théorie sous-jacente à chaque exercice par des définitions, des explications et des conseils aux enseignants sur le point de départ et le point d'arrivée de chaque exercice-, et ils avancent aussi l'application pratique de cette théorie, tout en donnant des exemples, soit du processus -où aller chercher les matériaux nécessaires pour, disons, composer une narration (diēgēma) ou une chrie ( chreia)-, soit du produit terminé, par exemple, en rédigeant une comparaison (synkrisis). Grâce à ces Traités nous avons sous les yeux une bonne partie de la phase finale du processus d'apprentissage auquel les jeunes étaient soumis pendant l'Empire et, vraisemblablement aussi à partir de l'époque hellénistique.

Il va sans dire que les auteurs des Traités expliquent la pragmatique de leur application, dans le but d'enseigner à produire diverses formes de discours, en tenant compte de la progression des difficultés, d'une part, et des exercices d'accompagnement, de l'autre. Voici comment Aélius Théon l'exprime ${ }^{6}$ :

Je n'ai pas fait cet exposé parce que je pensais que tout y convenait à tous les débutants, mais pour que nous sachions que l'entraînement à ces exercices est

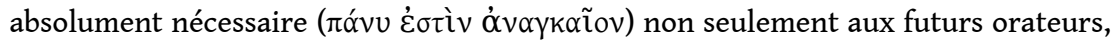
mais encore à tous ceux qui veulent pratiquer l'art des poètes, des historiens ou d'autres écrivains. En effet, ce sont là en quelque sorte les fondements de toute

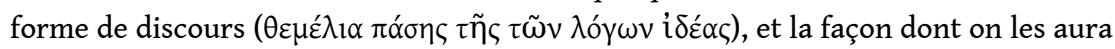

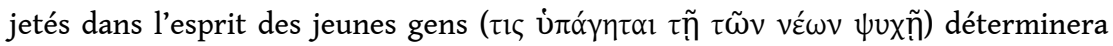
nécessairement la qualité aussi de la suite: aussi faut-il qu'en plus des exemples 
susdits le maître lui-même compose en particulier certaines contestations et

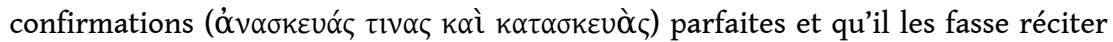
par les jeunes gens, afin qu'ils soient façonnés par la méthode de ces modèles

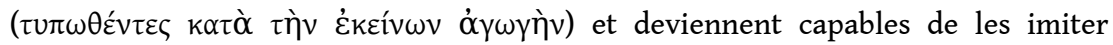
$(\mu \iota \mu \eta ́ \sigma \alpha \sigma \theta \alpha \iota)$.

6 Donc, si nous comprenons bien, les étudiants s'appliqueront à imiter un texte donné -en principe rédigé ou préparé par le maître- et mettront en œuvre leur talent en contestant et en confirmant les éléments constitutifs de celui-ci.

7 À côté de cela, et pour mieux exercer ce genre d'imitation, il faudra déployer trois autres

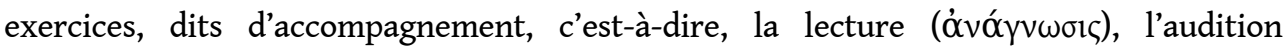

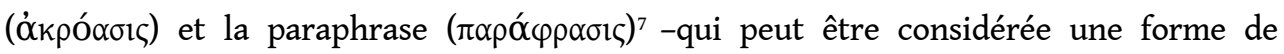
commentaire.

8 Les Traités de progymnasmata nous renseignent, d'une manière assez claire, à propos d'une démarche scolaire qui vise la formation rhétorique et littéraire. Or, le but envisagé -la formation rhétorique et littéraire- est atteint, à son tour, moyennant un certain nombre de pratiques lettrées, qui vont du niveau le plus bas -les débutants s'exerçaient à la flexion des cas et des nombres avec une chrie, par exemple- au niveau supérieur: la même chrie pouvait servir de base à la composition sur le mode de la contestation. De la même façon, un récit mythologique ou historique pouvait être le point de départ d'une composition sur le mode de la confirmation. En tout cas, il y a toujours un texte de base, qui est utilisé, manipulé, imité, contesté, confirmé.

De là, le passage naturel vers les formes littéraires paraît tout à fait évident. Prenons, par exemple, les discours siciliens d'Aelius Aristide (Or. 5 et 6): voilà deux pièces littéraires ayant la forme de discours politiques. En effet, dans ces deux discours du IIe siècle ap. J.C., deux orateurs fictifs prennent la parole, à l'assemblée d'Athènes, en 414 av. J.-C., pour répondre à Nicias qui se trouve en Sicile, et qui demande désespérément des renforts militaires ; le premier l'approuve, le second conteste ses propos. Le référent historique de cet épisode est évidemment un référent textuel : Thucydide 7.10-16. Aristide, à partir du contexte historique que le passage thucydidéen établit, façonne deux réponses au message de Nicias: il met en oeuvre, d'un côté la prosopopée -puisqu'il fait parler deux membres de l'assemblée athénienne-, et de l'autre, la contestation et la réfutation des propos du général athénien ${ }^{8}$.

Ce n'est qu'un exemple parmi d'autres: dans la plupart des textes en prose de l'époque impériale, la structure des progymnasmata est toujours répérable, au fond ou à la surface.

\section{Exercices scolaires d'après les textes latins}

11 En général, il faut commencer par affirmer que dans l'imaginaire romain, rien de ce qui a un rapport avec le ou les savoirs ne peut être indépendant de la culture grecque. Par conséquent, la culture grecque fait partie, d'une façon essentielle, de l'éducation des jeunes Romains. Or il faut avouer que, surtout avant l'Empire, lorsque les Romains s'expriment sur la formation du futur citoyen, il y a sans doute quelques différences qui sont parfois explicites et d'autres sous-entendues, mais qui relèvent de l'identité romaine par opposition à ce qu'ils entendent par identité grecque ${ }^{9}$. Cicéron, au début des Tusculanes en fait un synthèse parfaite, dont je souligne le passage suivant ${ }^{10}$ :

iam illa, quae natura, non litteris adsecuti sunt, neque cum Graecia neque ulla cum gente sunt conferenda. quae enim tanta gravitas, quae tanta constantia, magnitudo 
animi, probitas, fides, quae tam excellens in omni genere virtus in ullis fuit, ut sit cum maioribus nostris comparanda? Doctrina Graecia nos et omni litterarum genere superabat; in quo erat facile vincere non repugnantes. de rester fièrement romain ${ }^{11}$, et, pour y arriver, l'éducation est un élément clé: les jeunes Romains sont, en effet, élevés dans la paideia, mais, d'après les témoignages qui nous sont parvenus, la discussion porte surtout sur les dernières étapes scolaires, pour déterminer jusqu'où l'influence grecque doit arriver ${ }^{12}$. réaliser les coïncidences mais aussi les écarts par rapport à Aélius Théon, auteur d'un Traité de progymnasmata, qui était, vraisemblablement, son contemporain. Pour ce qui est des coïncidences ou, en tout cas, de la participation de Quintilien au système grec des progymnasmata, les livres 1 et 2 en témoignent largement ${ }^{13}$; il suit aussi, en général, dans les livres suivants, les théoriciens grecs de rhétorique; cependant, l'addition, à la fin, du livre 10, marque définitivement la différence; en effet, ce dernier livre de l'Institution Oratoire propose le tirocinium appliqué, c'est-à-dire, la mise en pratique réelle de tout ce qui a été appris, visant exclusivement la performance effective dans l'arène politique ou du barreau, ce qui, en fait, n'est pas envisagé dans les traités grecs ${ }^{14}$. Or, en pleine époque impériale, la vie politique ayant perdu, désormais, une très grande partie de son dynamisme délibératif, l'école est accusée de former les futurs orateurs avec des sujets toujours éloignés de la réalité, fictifs, qui n'aboutissent à rien du tout; c'est, justement, ce dont se plaignent les auteurs de l'époque impériale qui regrettent les temps, plus romains, où la préparation scolaire abordait des sujets de l'histoire de Rome ou des grands personnages du passé. Tacite, par exemple, ne saurait l'exprimer plus nettement ${ }^{15}$ :

nempe enim duo genera materiarum apud rhetoras tractantur, suasoriae et controversiae. ex his suasoriae quidem etsi tamquam plane leviores et minus prudentiae exigentes pueris delegantur, controversiae robustioribus ad signantur, quales, per fidem, et quam incredibiliter compositae! sequitur autem, ut materiae abhorrenti a veritate declamatio quoque adhibeatur. sic fit ut tyrannicidarum praemia aut vitiatarum electiones aut pestilentiae remedia aut incesta matrum aut quidquid in schola cotidie agitur, in foro vel raro vel numquam, ingentibus verbis persequantur: cum ad veros iudices ventum...

D'une façon ou d'une autre, pourtant, et malgré les écarts des manières strictement grecques de s'exercer à la rhétorique, surtout à l'époque républicaine, le noyau de la formation scolaire et de ses exercices demeure visible: l'essence formelle des textes produits hors des murs de l'école émane de la pratique de ces progymnasmata, évidemment d'un point de vue strictement formel de l'application et imitation ${ }^{16}$ des modèles, mais aussi -et ceci est, à mon avis, ce qui rapproche, en un sens, les progymnasmata de la pragmatique du commentaire- d'un point de vue structurel de la pensée.

\section{Cicéron et les progymnasmata}

15 En effet, une telle pratique scolaire entraîne, pour ainsi dire, une certaine façon d'organiser la pensée, même la réflexion la plus intime, et va au-delà, donc, d'une réalisation professionnelle concrète.

Je prends un texte de Cicéron comme exemple. Il s'agit d'une des lettres qu'il adresse à son ami Atticus. C'est à peu près le mois de mars de l'année 49, lorsque, aux débuts de la 
guerre civile, le sénateur Cicéron se voit dans la difficile situation de prendre parti, ou pour César, ou pour Pompée. Il s'exprime en ces termes-ci ${ }^{17}$ :

Ego etsi tam diu requiesco quam diu aut ad te scribo aut tuas litteras lego, tamen et ipse egeo argumento epistularum et tibi idem accidere certo scio; quae enim soluto animo familiariter scribi solent ea temporibus his excluduntur, quae autem sunt horum temporum ea iam contrivimus. sed tamen, ne me totum aegritudini dedam,

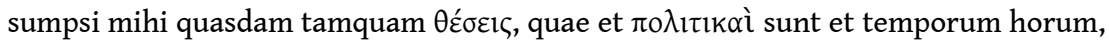
ut et abducam animum a querelis et in eo ipso de quo agitur exercear. eae sunt huius modi:

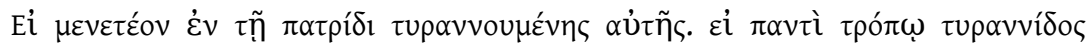

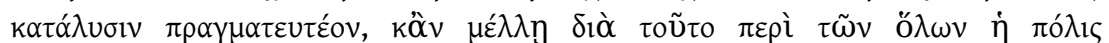

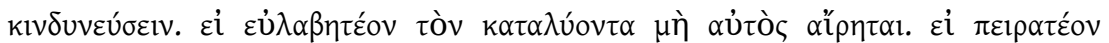

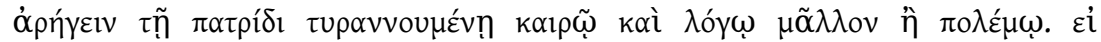

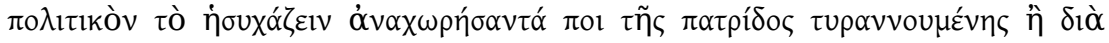

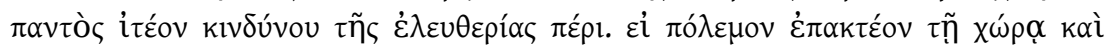

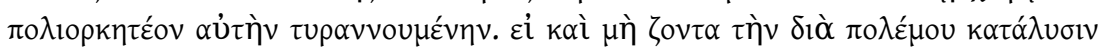

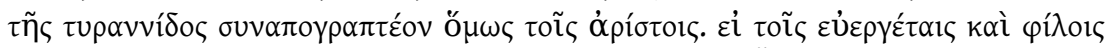

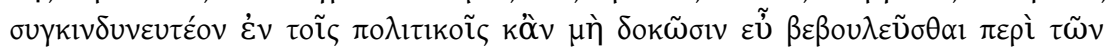

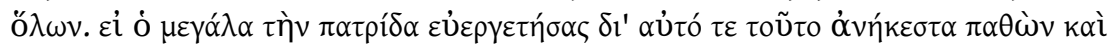

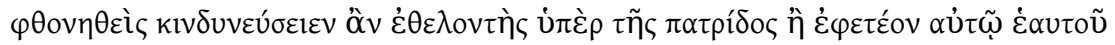

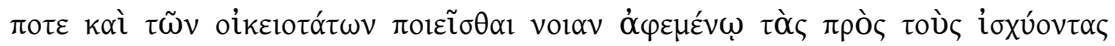

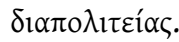

In his ego me consultationibus exercens et disserens in utramque partem tum Graece tum Latine et abduco parumper animum a molestiis et $\tau \tilde{\omega} v \pi \rho o u ̌ p y o v ~ \tau l$ delibero...

17 Je ne vais pas, évidemment, moi-même, faire le commentaire des allusions politicohistoriques du moment évoqué, ni des aspects autobiographiques de Cicéron qui se découvrent dans cette lettre. Je voudrais, en revanche, montrer comment la formation scolaire de Cicéron, et l'exercice des progymnasmata fait partie essentielle de sa façon, non seulement de communiquer avec son destinataire, mais de réfléchir et, somme toute, de penser, à un moment où, du point de vue politique et personnel, se joue pour lui une partie très importante.

En effet, dit-il, la matière lui manque, les circonstances sont tellement difficiles, et avancent si lentement, qu'il n'y a rien de nouveau à dire, sauf, évidemment, réfléchir, à haute voix en écrivant à un ami, sur le parti qu'il faut, faudra ou faudrait prendre. Pour ce faire, Cicéron se propose, dit-il, des $\theta \varepsilon ́ \sigma \varepsilon ı \varsigma$, et il emprunte le mot grec puisqu'il veut sans doute signifier le progymnasma qui porte ce nom: $\theta \dot{\varepsilon} \sigma \varsigma^{18}$. Ce que Cicéron entend dire, à mon avis, c'est qu'il reprend, d'une certaine façon, une activité scolaire, celle de s'exercer à un des progymnasta, la $\theta \dot{\varepsilon} \sigma ı \zeta$, pour réfléchir sur sa situation ${ }^{19}$.

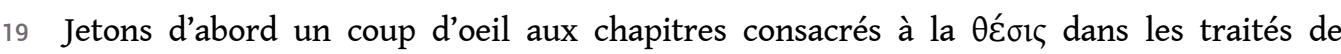
progymnasmata, ce qui nous aidera à comprendre la démarche de Cicéron; Théon dit $^{20}$ :

La thèse est un sujet qui comporte une controverse en paroles sans personnes définies ni circonstance aucune: Doit-on se marier? Doit-on avoir des enfants? Les dieux existent-ils?

$[\ldots]$

Soit, à titre d'exemple, une première thèse pratique: un sage se mêlera-t-il de gouverner? Pour confirmer qu'il doit gouverner, on dira...

Les technographes ont défini la thèse comme l'examen, sans qu'intervienne aucune circonstance particulière, d'un sujet de discussion. La thèse en effet semble se 
ramener à une délibération de caractère général, parce qu'elle règle son déroulement, non pas par rapport à une personne donnée, mais simplement et d'une manière générale, par rapport à toute personne et par la seule considération des implications du sujet. En effet, lorsque nous examinons si c'est un devoir de prendre femme (...) nous considérons le sujet en lui-même et nous faisons l'examen de ses implications: une personne quelconque doit faire ceci, attendu qu'il en résulte telles conséquences pour ceux qui le font?

Et finalement, Aphthonios ${ }^{22}$ :

La thèse est l'examen en paroles d'un sujet de discussion. Les thèses sont politiques ou théoriques. Sont politiques celles qui envisagent une action utile au salut de la cité, par exemple: faut-il se marier? Faut-il être marin? Faut-il construire des remparts? (...) Sont théoriques celles dont la discussion n'engage que l'intellect, par exemple: le ciel est-il sphérique? Y a-t-il plusieurs mondes? En effet cela n'a pas d'incidence sur la vie pratique, mais est un débat purement intellectuel.

\section{Du côté romain, c'est Quintilien qui ébauche aussi une définition de $\theta \varepsilon ́ \sigma ı \varsigma^{23}$ :}

Thesis autem quae sumuntur ex rerum comparatione (ut 'rusticane uita an urbana potior', 'iuris periti an militaris uiri laus maior') mire sunt ad exercitationem dicendi speciosae atque uberes, quae uel ad suadendi officium uel etiam ad iudiciorum disceptationem iuuant plurimum: nam posterior ex praedictis locus in causa Murenae copiosissime a Cicerone tractatur. Sunt et illae paene totae ad deliberatiuum pertinentes genus: 'ducendane uxor', 'petendine sint magistratus'; namque et hae personis modo adiectis suasoriae erunt.

3 Tous les auteurs s'accordent sur le fait que la $\theta \varepsilon ́ \sigma ı \varsigma$ ne doit évoquer ni une situation ni un personnage concrets; la seule différence -que chacun à sa façon apporte- réside dans le but pratique ou spéculatif de la question posée.

L'exemple de thèse pratique de Théon, en plus, touche le même sujet que les thèses de Cicéron; la proximité du sujet, donc, révèle d'une façon plus claire si possible la nature progymnasmatique des réflexions de Cicéron; en effet, il est question, si nous continuons la lecture de Théon là où nous l'avons laissée, d'arguments multiples, parmi lesquels certains sont à mettre en rapport avec les thèses de Cicéron, par exemple ${ }^{24}$ :

[le sage se mêlera-t-il de gouverner]

«même si cela ne lui était pas facile, du moins doit-on faire passer le bonheur de la Cité avant ses propres peines ».

"le gouvernement est approprié au sage: qui mieux que lui rendra la justice, délibérera de ce qui est opportun, proposera lois et décrets, sans se laisser corrompre dans l'exercice de toutes ces fonctions? »

«il est juste de payer de retour sa patrie, ses concitoyens et les dieux de ses pères, de qui on a reçu la nourriture et l'instruction ».

«une forme de gouvernement qu'on néglige et qui évolue en mal est diffcile à restaurer et celui qui, avant qu'elle devienne mauvaise, s'est montré négligent en éprouve du regret à un moment où cela ne sert plus à rien ".

Il y a trois aspects qui intéressent mon propos dans cet exemple. Le premier est évident: Cicéron exerce sa pensée au moyen de ce qui était une pratique scolaire, en principe complètement détachée de la vie quotidienne: il s'agissait, comme nous l'indiquent les auteurs des Traités, de la manière d'aborder un sujet quelconque, la plupart du temps issu des matières traitées par la tradition philosophique, littéraire, politique, et que l'on pouvait trouver dans les ouvrages des anciens. Et ceci avait pour but, non pas d'apprendre à vivre, ou à prendre des décisions personnelles importantes, mais de construire un discours. 
26 Le deuxième aspect, un peu plus compliqué, montre, encore plus que le précédent, comment l'école et la pragmatique des exercices d'école font partie désormais de la structure de la pensée des individus qui s'y sont formés. En effet, en proposant les $\theta \dot{\sigma} \sigma \varepsilon 1 \varsigma$ que nous venons de lire, Cicéron trouve le moyen de se détacher lui-même du problème personnel qui l'affecte, et essaie, par ce détachement, de le résoudre.

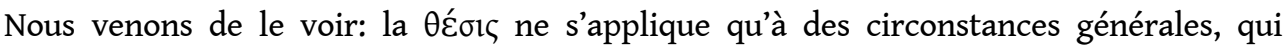
n'impliquent pas des individus -pour cela il y a d'autres exercices: le lieu commun, la

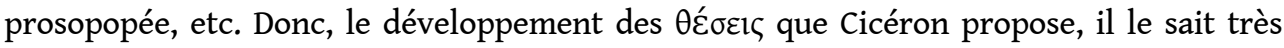
bien, même s'il n'arrive pas à les développer effectivement, apportera des éléments externes à son ennui présent -par exemple, des prises de position pour ou contre, la nécessité, la noblesse, le profit, le plaisir, relatifs au sujet annoncé, ou bien s'il est d'accord avec la nature et les mœurs, s'il est aisé de faire l'un ou l'autre, si d'autres, dans le passé, ont pris cette décision ou la décision contraire, si elles furent justes, honorables, méritoires, dignes des grands moments et des grandes occasions, si cela était en accord avec les ancêtres et la volonté divine, etc. Or, moyennant le détachement du problème

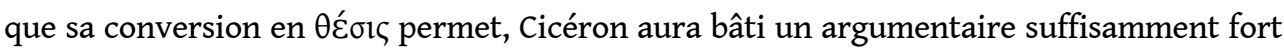
pour justifier, pour lui-même et pour les autres, n'importe laquelle des décisions qu'il pourra prendre.

Ceci, évidemment, du point de vue de sa prise de décision personnelle: l'objectivation de son expérience à travers les thèses le rassurera puisqu'il aura pu traiter le problème en éloignant sa subjectivité, en éliminant ses émotions; comme l'avocat au barreau, il restera calme et n'aura que la raison comme guide, il aura réussi à garder le contrôle de la situation comme s'il s'agissait d'une chose détachée de lui-même ${ }^{25}$.

Et troisièmement, il y a encore un autre avantage à expliquer ainsi à son ami Atticus son état d'esprit: finalement, les thèses ne sont qu'une façon, oblique certainement, de décrire la réalité telle que Cicéron la ressent; par conséquent, Atticus peut très bien comprendre qui est le tyran et la tyrannie dont il est question, il peut aussi imaginer tout de suite quelle cité Cicéron évoque lorsqu'il parle de « ruine totale de la cité », et lorsqu'il parle de guerre, ce n'est bien sûr pas à une guerre quelconque que l'on songe, ainsi que quand la liberté est nommée, ou "les citoyens du bon parti », ou les «bienfaiteurs et amis »; et finalement, lorsqu'il est question, dans la dernière des thèses de l' " homme qui a rendu à la patrie des signalés services, etc. », Atticus et quiconque lira cette lettre comprendra très bien que c'est de lui-même que l'auteur de la lettre parle. Il ne pourra, pourtant, pas être accusé de sédition ni d'autres trahisons politiques puisqu'en réalité cette description si nette de la situation du pouvoir à Rome en ce moment n'est, à la limite, qu'allusive; formellement ce ne sont que des exercices scolaires, des $\theta \dot{\sigma} \sigma \varepsilon ı \zeta$. Justement, en tant que

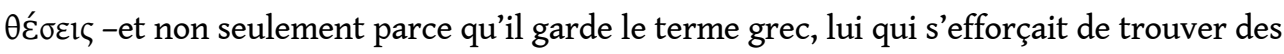
équivalents en latin ${ }^{26}$ - ce ne sont pas les realia qu'elles évoquent, mais l'univers, fictif, référentiel, livresque, typifié, lointain, de la paideia grecque.

L'exemple de Cicéron n'est pas, bien sûr, le seul que nous pouvons évoquer; l'expression, à l'abri des progymnasmata, des critiques au pouvoir est un outil très employé par la ruse

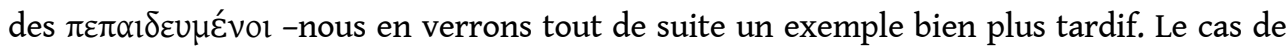
Cicéron, cependant ajoute un intérêt supplémentaire: il est un homme fort du dernier moment de la République Romaine, le temps où l'opposition entre une formation rhétorique de répertoire standard (modèle grec) et la préparation pour l'exercice automatique du métier d'orateur (modèle romain) est sans doute bien significative. Son 
retour, pour ainsi dire, au plus typique de l'école grecque est à la fois, dans sa circonstance compliquée, un refuge spirituel, une protection politique et un alibi juridique.

\section{Progymnasmata de Libanios et l'autorité d'Homère}

31 Or, si nous prenons un auteur plus tardif -et nous embrasserons ainsi presque tout l'arc temporel de la pragmatique scolaire que nous connaissons-, le sophiste ami de l'empereur Julien, Libanios (IVe ap. J.-C.), nous verrons que la démarche est similaire. Libanios, lui, nous a légué un bon nombre de ses compositions, d'une part, les compositions scolaires -nous conservons ses progymnasmata et ses déclamations-, et de l'autre, ses discours politiques et ses lettres. Bernard Schouler a montré comment ils exercent une influence réciproque les uns sur les autres; par exemple, son Éloge de Thersite (Lib. Enc. 8.4), chef-d'oeuvre scolaire du progymnasma $\dot{\gamma} \gamma \omega ́ \mu$ iov, qui ajoute l'élément paradoxal et la critique d'Homère à l'apparente platitude de l'exercice, sera pour le sophiste d'Antioche l'occasion de mettre en valeur la vertu de la parrhēsia, qui à son avis, est fondamentale devant l'autorité; le parallélisme est clair: dans l'Éloge de Thersite, c'est l'autorité d'Homère, tout d'abord, ainsi que celle d'Agamemnon et des chefs achéens, qui sont contestées par la parrhēsia de Thersite; tandis que, par exemple, dans le discours Contre ceux qui l'avaient appelé inopportun (Lib. Or., 2), Libanios offre -et j'emprunte l'expression à Schouler- « un excellent témoignage de la parrhēsia du sophiste » (schOULER 2013:241): en effet, postérieur de deux ans à l'avènement de Théodose, le grand empereur chrétien de la fin du IVe siècle, ce discours de Libanios est un programme politique incisif contre son gouvernement.

De Cicéron à Libanios la distance est énorme : d'abord, distance temporelle, sans doute, mais aussi deux sortes de distances socio-culturelles: d'abord, celle qui écarte le monde de la dernière République romaine, avec l'action politique au premier rang, de la tradition grecque ininterrompue, avec tout le poids de la Seconde Sophistique et ses rapports avec le pouvoir impérial ; et après, l'arrière-fond d'un vrai changement de modèle culturel promu par le pouvoir impérial lui-même. Cependant, l'empreinte scolaire des progymnasmata comme outils d'expression, de description, de commentaire voilé de la réalité, politique dans les deux cas, subsiste.

Pratiques scolaires ou non scolaires, le fait est que la pragmatique générée par les progymnasmata scolaires étend un manteau bien épais sur un bon nombre de pratiques textuelles de l'antiquité gréco-romaine, et en détermine aussi une idéologie intellectuelle, fondée sur une constatation apparemment paradoxale: la nature absolument rigide du système d'éducation donne lieu à des pratiques intellectuelles tout à fait ouvertes, à une grande capacité de création à partir de l'imitation, et, somme toute, à une pensée richement versatile. 


\section{Éditions et traductions des traités de Progymnasmata}

KENNEDY George A., Progymnasmata. Greek Textbooks of Prose Composition and Rhetoric, Atlanta, SBL, 2003.

PATILLON Michel, Aelius Théon. Progymnasmata, Paris, CUF, 1997.

PATILLON Michel, Corpus rhetoricum, Paris, CUF, 2008.

RABE Hugo, Hermogenis opera, Leipzig,Teubner, 1913 (repr. Stuttgart, 1969).

RABE Hugo, Aphthonii progymnasmata, Leipzig, Teubner, 1926.

SPENGEL Leonhard von, Rhetores Graeci, vol. 2., Leipzig, Teubner, 1854.

\section{Ouvrages cités}

BOMPAIRE Jacques, « Les historiens classiques dans les exercices préparatoires de rhétorique (progymnasmata) », in Recueil Plassart: Études sur l'antiquité grecque offertes à André Plassart par ses collègues de la Sorbonne, Paris, Les Belles Lettres, 1976, p. 1-7.

BONNER Stanley F., Education in Ancient Rome: From the Elder Cato to the Younger Pliny, London, Methuen, 1977.

BONNER Stanley F., Roman Declamation in the Late Republic and Early Empire, Liverpool, University Press, 1949.

BOUTIN Renaud, «Quand Demosthène parle latin. Le rôle des orateurs grecs dans la définition cicéronienne de l'éloquence ", in DUPONT Florence \& VALETTE-CAGNAC Emmanuelle (dir.), Façons de parler grec à Rome, Paris, Belin, 2005. p. 135-174.

CELENTANO Maria Silvana, « L'oratore improvvisa. A proposito di Quintiliano, InstitutioOratoria 10.7 », in PETRONE Gianna \& CASAMENTO Alfredo (dir.), Studi... in umbra educata. Percorsi della retorica latina in età imperiale, Palermo, Flaccovio, 2010, p. 141-160.

CLARKE Martin L., Higher Education in the Ancient World, London, Routledge \& Kegan, 1971.

CLARKE Martin L., " The Thesis in the Roman Rhetorical Schools of the Republic », Classical Quarterly,1.3-4, 1951, p. 159-166.

CRIBIORE Raffaella, Gymnastics of the mind : Greek education in Hellenistic and Roman, Princeton. N.J., Princeton University Press, 2001.

CRIBIORE Raffaella, Writing, teachers, and students in Graeco-Roman Egypt, Atlanta, Scholars Press, 1996. 
CULPEPPER STROUP Sarah, « Greek Rhetoric meets Rome: expansion, resistance, and acculturation », in DOMINIK William \& HALL Jon (dir.), A Companion to Roman Rhetoric, Malden \& Oxford, Blackwell, 2007, p. 23-37.

KASTER Robert A., "Controlling reason: declamation in rhetorical education at Rome ", in TOo Yun Lee (dir.), Education in Greek and Roman antiquity, Leiden \& Boston, Brill, 2001, p. 317-337.

KENNEDY George A., "Some recent controversies in the study of later Greek Rhetoric ", The American Journal of Philology,124.2, 2003, p. 295-301.

KENNEDY George A., The art of rhetoric in the Roman world, 300 B.C.-A.D. 300, Princeton, N.J., Princeton University Press, 1972.

MCNELIS Charles, « Greek Grammarians and Roman Society during the Early Empire: Statius' Father and his Contemporaries », Classical Antiquity, 21.1, 2002, p. 67-94.

MARRou Henri-Irénée, Histoire de l'éducation dans l'Antiquité. Paris, Seuil, 1948.

MESTRE Francesca, «Eli Arístides: Entre l'imperi atenès i la pax romana (Or. V-VI K i XI-XV K) », Ítaca, 2, 1986, p. 107-130.

MORGAN Teresa, Literate education in the Hellenistic and Roman worlds, Cambridge \& New York, Cambridge University Press, 1998.

PENELLA Robert J., « The Progymnasmata in Imperial Greek Education », Classical World,105.1, 2011, p. 77-90.

PERNOT Laurent, La rhétorique de l'éloge dans le monde gréco-romain, 2 vols., Paris, Institut d'Études Augustiniennes, 1993.

PERNOT Laurent, Les Discours siciliens d'Aelius Aristide (Or. 5-6), New York: Arno Press, 1981.

SCHOULER Bernard, « Libanios ou la sophistique comme critique », in MESTRE Francesca \& GÓMEZ Pilar (dir.), Three centuries of Greek culture under the Roman Empire, Barcelona, UBe, 2014, p. 229-250.

VIX Jean-Luc, L'enseignement de la rhétorique au IIe siècle ap. J.-C. à travers les discours 30-34 d'Aelius Aristide, Turnhout, Brepols, 2010.

WEBB Ruth, Ekphrasis, Imagination and Persuasion in Ancient Rhetorical Theory and Practice, Farnham \& Burlington, Ashgate, 2009.

WEBB Ruth, « The Progymnasmata as practice », in TOO Yun Lee (dir.), Education in Greek and Roman antiquity, Leiden \& Boston, Brill, 2001, p. 289-316.

WINTERBOTTOM Michael, « Schoolroom and courtroom », in VICKERS, Brian (dir.), Rhetoric Revalued. Papers for the International Society for the History of Rhetoric. Binghamton, N.Y., Center for Medieval \& Early Renaissance Studies, 1982, p. 59-70.

\section{NOTES}

1. MORGAN, 1998; CRIBIORE, 1996 et 2001.

2. Sur l'origine et développement des exercices, cf. VIX, 2010:333; PERNOT, 1993: 1.58.

3. Quint. Inst. 2; Rhet. Her., Sen. Contr.; Suet. Rhet.

4. KENNEDY, 1972; BOMPAIRE, 1976; CRIBIORE, 2001; WEBB, 2001 et 2009 ; PENELLA, 2011.

5. KENNEDY, 2003: 295. 
6. Theon Prog. 70.24-71.1 (édition et traduction PATILLON, 1997).

7. ibidem $61.30-33 ; 62.1-10 ; 62.10-20$.

8. Cf. PERNOT, 1981; MESTRE, 1986.

9. Même si ce schéma grec/romain est bien sûr très simpliste pour décrire la réalité de ces sentiments identitaires, on ne peut pas nier que la dichotomie nous/eux est souvent évoquée.

10. Cic. Tusc. 1.2-3, trad. Humbert (CUF 1931): «Et maintenant, lorsqu'on en vient aux avantages que donne le caractère et non l'étude, ni les Grecs, ni d'ailleurs aucun autre peuple ne peut nous être comparé. Où trouver un degré de dignité, un degré de fermeté, de grandeur d'âme, de probité, de loyauté, où trouver, dis-je, chez aucun peuple, une supériorité de mérite à tous les points de vue telle qu'on puisse le mettre en parallèle avec nos aïeux? Sous le rapport de la culture générale, il est vrai, et dans tous les genres littéraires, les Grecs l'emportaient sur nous; mais sur ce terrain, il leur était facile de remporter une victoire qu'on ne leur disputait pas. »

11. Cf. BOUTIN, 2005.

12. La bibliographie sur les interactions entre le modèle grec et le modèle romain -modèles civiques, politiques, et, donc, scolaires- est énorme; je donne seulement deux titres qui me semblent pertinents en ce qui concerne l'éducation des dernières années de la République et le premier Empire: CULPEPPER STROUP, 2007; MCNELIS, 2002; sur l'éducation romaine en général, cf. KENNEDY, 1972; BONNER, 1977; WINTERBOTTOM, 1982 ; CLARKE, 1971.

13. À propos des exercices qui sont à faire chez le grammaticus ou chez le rhetōr, cf. 1.9; pour la description des exercices, cf. 2.4 .

14. Cf., sur le livre 10 de Quintilien, CELENTANo, 2010; cf. aussi, sur l'école rhétorique romaine de la République ne visant qu'un futur métier politique ou d'avocat de l'étudiant, BONNER, 1949.

15. Tac. dial. 35.4-5, trad. Bornecque (CUF 1960): « En effet, si je ne me trompe, on traite chez les rhéteurs des sujets de deux espèces: les suasoriae et les controverses. Les suasoriae ont beau être abandonnées aux enfants, comme beaucoup plus faciles et exigeant moins de pratique, les controverses être réservées aux élèves plus âgés; quels sujets, grands dieux! quelles hypothèses incroyables. De plus, ces sujets, qui n'ont aucun point de contact avec la réalité, sont développés d'un style déclamatoire. Voilà comment ce sont les tyrannicides, l'alternative laissée aux jeunes filles violées, les remèdes à la peste, l'inceste des fils avec leur mère, et autres sujets agités chaque jour à l'école, au forum rarement vus jamais, qui sont traités d'un ton emphatique. Mais devant un vrai tribunal...»

16. Sur le sens d' « imitation » traduisant le terme imitatio latin, cf. BOUTIER 2005: 139-142.

17. Cic. Att. 9.4, trad. Bayet (CUF 1964): «Je n'ai repos d'esprit qu'aux moments où je t'écris ou lis tes lettres; mais la matière me manque, et à toi aussi, je le sais bien: les propos familiers auxquels engage la liberté d'esprit sont exclus par les circonstances, et nos échanges sur celles-ci sont usés jusqu'à la corde. Pour éviter cependant de m'abandonner tout entier à l'inquiétude, je me propose des sortes de thèmes, à la fois politiques et liés aux circonstances actuelles propres à distraire mon esprit de ses plaintes et à l'exercer sur l'objet même qui nous occupe. En voici le genre: Si l'on doit rester dans sa patrie tombée sous la puissance d'un tyran?Si l'on doit par tous moyens poursuivre la destruction de la tyrannie au risque d'une ruine totale de la cité?S'il faut prendre garde que le libérateur ne s'érige pas lui-même en tyran?S'il ne faut pas préférer à la guerre, pour aider sa patrie contre la tyrannie, l'opportunité et les voies de raison?Si la conscience du devoir politique permet, en période de tyrannie, de se retirer en quelque retraite, ou s'il faut aller à travers les plus grands périls à la reconquête de la liberté?S'il convient de porter la guerre contre son pays et de le mettre en état de siège, parce qu'un tyran l'occupe?Si, même quand on n'approuve pas une libération par les armes, il faut s'engager en ce sens avec les citoyens $d u$ bon parti?S'il faut partager les périls de ses bienfaiteurs et amis pendant la crise politique, alors qu'ils vous paraissent avoir accumulé les erreurs en toutes circonstances?Si un homme qui a rendu à sa patrie de signalés services et qui, pour cette raison même, a été victime de traitements et de jalousies intolérables, a à s'exposer volontairement pour sa patrie, ou si on doit lui permettre d'avoir égard 
à lui-même et à ses plus proches en laissant aux puissants leurs différends politiques?Telles sont les discussions où je m'exerce, débattant le pour et le contre en grec et en latin. Cela distrait tant soit peu mon esprit de ses chagrins et je précise utilement cet aspect des problèmes. » (en italiques les parties en grec dans le texte de Cicéron).

18. Ni la traduction "thèmes", ni la note explicative en bas de page dans l'édition de J. Bayet ("terme technique signifiant: questions posées de manière générale ») ne recouvrent malheureusement pas le vrai sens de l'expression. D'autre part, les Romains emploient d'habitude le mot grec pour nommer cet exercice, n'ayant trouvé de traduction convenable, malgré les efforts de Cicéron, cf. Cic. inv. 1.8; orat. 2.78.

19. Sur la Өદ́бıৎ dans les écoles romaines à l'époque de la République, cf. Clarke 1951.

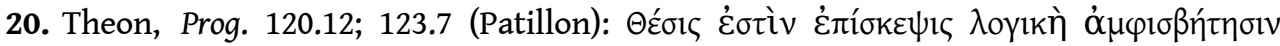

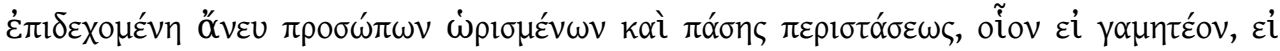

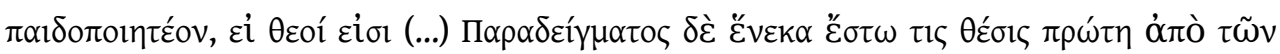

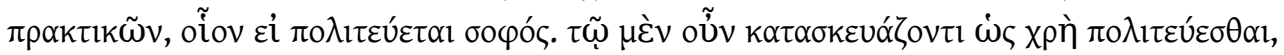

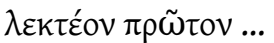

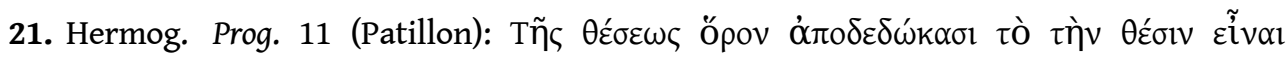

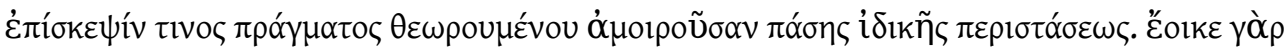

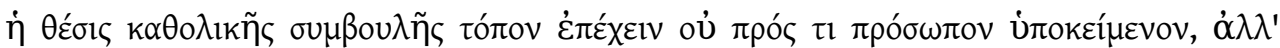

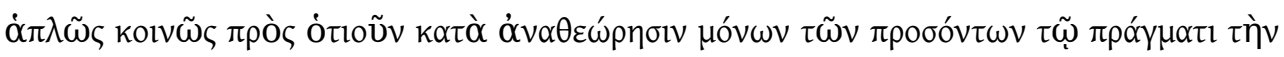

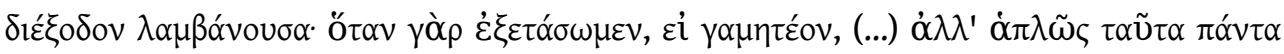

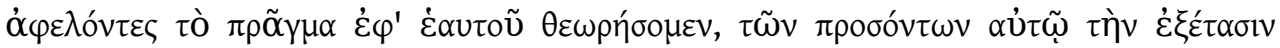

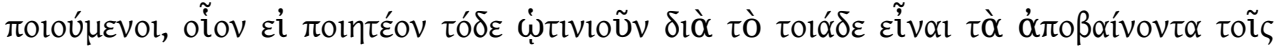
$\mu \varepsilon \tau \iota$ ป̃ $\sigma \mathrm{lv}$.

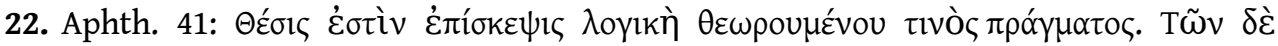

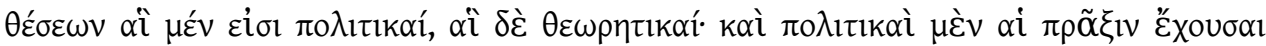

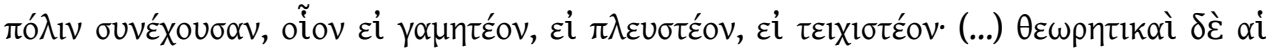

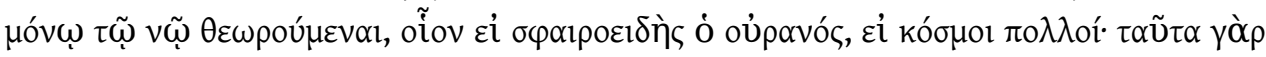

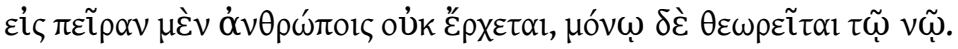

23. Quint. Inst. 2.4.24, trad. Cousin (CUF 1976): «Et les thèses? Elles s'inspirent de la comparaison d'une chose avec une autre (par exemple, "si la vie de la campagne est préférable à celle des villes?" ou "si le renom d'un jurisconsulte l'emporte sur celui d'un militaire?") Pour exercer à la parole, les thèses ont étonnamment d'attrait et de richesse et, qu'il s'agisse de persuader ou même de débattre dans un procès, elles aident beaucoup. Aussi bien le second des sujets ci-dessus est-il très largement traité par Cicéron dans le procès de Muréna. Il y a aussi des thèses qui se rapportent presque uniquement au genre délibératif: "Faut-il prendre femme? Briguer les charges publiques?" En effet, il suffit que l'on y insère des noms de personnes, et ce seront de suasoires».

24. Théon 123.26-124-17.

25. Cf., dans ce sens, KASTER, 2001.

26. Cf. supra n. 16 . 


\section{RÉSUMÉS}

Les pratiques lettrées d'une civilisation reposent toujours, d'une façon ou d'une autre, sur le système scolaire de cette civilisation. Dans le système scolaire grec, adopté aussi par les Romains au moins à partir du IIe siècle av. J.-C., les exercices scolaires, visant un but utilitaire, c'est-à dire, surtout la préparation de futurs hommes forts du pouvoir politique, sont un outil précieux pour le grammatikos et pour le rhetör, et pour leurs disciples en formation. Ces exercices ou progymnasmata, exactement comme d'autres pratiques telles que le commentaire, le discours d'apparat et nombre d'autres productions textuelles, reposent presque exclusivement sur l'interprétation métatextuelle de la tradition. Mon but dans ce travail est de montrer le passage de la démarche scolaire à l'expression de la pensée hors les murs de l'école. En effet, la référence, l'évocation et la paraphrase continuelles de cette tradition deviennent, à leur tour, un instrument très utile, puisque celui-ci permet un certain détachement émotionnel, et très naturel -et, par conséquent, juste, non nocif, prestigieux- pour décrire, interpréter, communiquer, attaquer certains aspects de la réalité. Il sera intéressant de noter, aussi, que cette pratique demeure toujours utile et profitable tout au long de l'Antiquité.

Literate practices of a civilization are based, in one way or another, on the school system of this civilization. Within the Greek educational system, adopted by the Romans at least from the second century BC., school exercises, targeting a utilitarian purpose -that is to say, especially the preparation of future politicians- are a valuable tool both for grammatikoi and rhetores, and for their disciples as well. These exercises, called progymnasmata, consist almost exclusively in the metatextual interpretation of tradition, just as other practices such as commentary, speeches and many other textual productions. My goal in this work is to show the transition from school approach to the expression of thought outside the walls of the school. Indeed, the reference, evocation and continuous paraphrase of tradition become, in turn, very useful strategies to get emotional detachment, and a natural way -and, therefore, fair, not harmful, prestigious- to describe, interpret, communicate, and address certain aspects of reality. It will be interesting to note, too, that this practice is still useful and profitable throughout antiquity.

\section{INDEX}

Mots-clés : Progymnasmata, éducation grecque, thesis, Cicéron, Libanios

Keywords : Progymnasmata, Greek Education, Thesis, Cicero, Libanius

\section{AUTEUR}

\section{FRANCESCA MESTRE}

Professeur de Philologie Grecque à l'Université de Barcelone. Ses travaux de recherche et ses publications se concentrent presque exclusivement sur les écrivains de l'époque impériale (Plutarque, Dion Chrysostome, Lucien, Philostrate, dont elle est aussi éditrice et traductrice) et, 
surtout, les dernières années, sur les phénomènes culturels que la cohabitation de grecs et romains, païens et chrétiens, hellénophones et latinophones façonne au sein de l'Empire Romain.Elle dirige le groupe de recherche Graecia Capta. Fmestre[at]ub.edu 